\title{
The Application of Simulation Analysis in the Screw Casting of Screw Expanding Generators
}

\author{
Zhixin Chen ${ }^{1}$, Wenwei Zhuang ${ }^{1}$, Sufen Yan $^{2}$, Haiying Zhou ${ }^{1}$ \\ ${ }^{1}$ School of Mechanical and Electronic Engineering, East China university of Technology, Nanchang, Jiangxi, China \\ ${ }^{2}$ Jiangxi Institute of Computing Technology, Nanchang, Jiangxi, China
}

\begin{abstract}
The screw expanding generator is a kind of low-grade thermal energy machines which has a wide application future. The production and processing of key part - screw is vital. To improve products quality, this paper uses the advanced simulation analysis to state the production and male screws processing, the principle of simulation analysis and the use of related software in detail with taking a case of male screw processing. It optimizes the design of male screw processing (mainly riser casting choice), and gets a better one. This paper offers a corresponding solution for this kind of process problems.
\end{abstract}

\section{Introduction}

The screw expanding power generator is a kind of lowgrade thermal energy machine. Its construction includes two screw rotors (male and female) screw rotors, a shell, bearings, sealing devices and so on. When the screw expanding power generator works, it can recycle lowgrade waste heat and steam, steam-water-mixture, flue gas, geothermal and other low-grade heat sources. It can make steam into screw tooth space through an air inlet port. And then the steam drives the screw rotation. It decreases pressure and temperature of steam, makes the steam work, goes out from an exhaust port and exports from mainshaft screw. As a prime mover driving generator working and changing low-grade heat sources into power, it is a breakthrough in the area of renewable resources utilization.

\section{Simulation Technology}

Due to the size of male screw is larger, practicality experiments of the equal sizes are impossible. And experiments of small pieces sometimes differ from practical production process too much. At the same time, physical experiments usually only give a result of a certain stage in the manufacturing process, not a complete picture of the entire manufacturing process. There are some limitations. However, the numerical simulation technology on casting process can change production factors in filling and solidification processes into the counting factor which is made by computer simulation. It can reflect the filling and solidification processes of casting and predict accurately positions where defects appear, their sizes and so on in castings by visualization methods. Owing to these specificities of production of large size parts, the use of advanced numerical simulation technique in process has significant meaning that change the recent situation which is only decided by experience in process.

This paper chooses large size parts- male screws (mainly about riser design) to of process design and computer simulation.

\subsection{Flow field simulation and temperature field simulation of mold filling processes}

The liquid metal flow in mold filling processes follows the law of fluid dynamics. It can be described by basic governing equations of mass momentum conservation. Liquid metal and cast in mold filling process can be described by heat exchange between establishments of the heat balance equation. The above problems can be solved by several numerical methods. The most common way is the SO-LA-VOF method. In the numerical simulation of mold filling process, the liquid metal is treated as incompressible fluid; its flow process follows conservation of mass and momentum conservation. Its mathematical versions are equation of continuity and the Navier-Stokes equation (N-S equation).

\subsection{The temperature field simulation of casting solidification process}

Solidification is a very complex physical and chemical process. It is coupled by heat transfer process, momentum transfer process, mass transfer process, phase change process and other processes. To simulate the solidification process, it accurately must solve the equation of continuity, Navier-Stokes equation, Fourier equation and mass transfer equation. 
The casting / mold heat transfer system processes proceed through three comprehensive way which are radiation heat transfer of heat metal, transfer between liquid metal and the cast heat (including convection heat between casting mold surface and atmospheric heat), and metal casting heat conduction. These three kinds of heat transfer methods established corresponding mathematical models.

\subsection{The Value of Simulation Analysis}

Casting process is a thermoforming process that pours liquid metal into a closed mould cavity, and then obtains required shape castings through the filling and solidification processes. In the whole process, a mutual coupling among a velocity field, a stress field and a temperature field is very complex. It is difficult to get any scientific results by real-time observation and measurement. Therefore, the use of computers that to simulate casting filling and solidification processes numerically is very necessary. From the 1960 's, the solidification process began to simulate numerically. In the early 80's the filling process and stress-strain of castings began to simulate numerically .And in the 90's, studies of microstructure of castings began to simulate numerically. The numerical simulation technique has gone deep to all aspects of processes of casting forming. Studies of simulation are developing to microstructure simulation, performance optimization and predictions of useful life. It becomes a multifunctional, high fidelity, and high efficient multi-disciplinary simulation technique. According to the determination of National Institute of Science and Technology, qualities of products can be raised 5 to 15 times by the computer simulation, production yield can be raised $25 \%$, production cost can be reduced $13 \%$ to $30 \%$, labor cost can be reduced $5 \%$ to $20 \%$, capacity utilization can be increased to $30 \%$ to $30 \%$, and design and trial period can be shortened $30 \%$ to $60 \%$. Thus, the potential of the computer simulation technique of materials processing is huge in the field of materials research.

The computer numerical simulation in casting process includes the temperature field numerical simulation in the solidification process, flow field numerical simulation in the filling process, stress field numerical simulation and microstructure morphology numerical simulation. Through studying the numerical simulation of these single and coupled processes, you can analyze some casting quality problems such as shrinkage, shrinkage porosity, gas hole, slag, misrun, crack, and other problems which appear in the cast process. Then you can find out internal reasons that problems happen, and finally optimize the casting process, eliminate casting defects and improve the qualities of products. The quality of processes can be predicted by casting simulation software before production. Compare different process plans, and achieve process optimization. As the product quality prediction can be done on computer and produce actually, it can save a lot of labor, material and financial resources. Especially to casts from trial production of new products and mass production molding lines, it is not only convenient, but also has significant economic beneficiation. Numerical simulation can also change the old casting process designs which have established on the basis of experience into science.

\section{The Software Introduction}

In recent decades, some corresponding commercial software about analyzing casting formation process appeared continuously with the constant improvement and perfection of numerical simulation technique in cast filling and solidification process. The development of the first software about this aspect is led by Sahm PR, a professor in University of Aachen, Germany. It is called MAGMA Soft in 1989. After that a lot of commercial softwares continue to emerge. Nowadays, many countries have their own commercial simulation softwares, such as AFS Solidification System, Flow3D, ProCAST (the United States), SIMULOR (France), SOL STAR(the UK), ADSTEFAN(Japan) and so on. These softwares have been combined with the actual production. They are widely used in casting enterprises of developed countries and have achieved good economic benefits. Some of them have been used in China's large enterprises and research institutes.

Although these softwares have their own characteristics, they can basically complete filling process analysis, solidification process analysis, residual stress analysis, deformation analysis, prediction about defects and performance of casting analysis that under various casting alloys which include sand mould casting and a variety of casting methods. In addition, some softwares can analyze microstructure of casting at the same time, that is a future direction for casting engineering developing.

In this paper, the analysis software for FLOW-3D is three-dimensional engineering fluid dynamics software, suitable for simulation of liquid metal flow and solidification processes.

FLOW-3D, analytic software applied for threedimensional engineering fluid mechanics which this paper use, is suitable for simulation of liquid metal flow and solidification process.

FLOW-3D, officially launched in 1985, is developed by an internationally renowned master hydrodynamics Dr. CW Hirt. It earned a good reputation for its precise calculation result and accurate simulation of practical problem and calculating skills of CFD (Computational Fluid Dynamics). Its special technique--FAVOR, provides more accurate and efficient solutions for complicated issues focused on free surface, as to metal casting.

\section{Case Analysis}

To use Pro / E to draw the screw, to save it as STL format and then to enter Flow-3D for analysis (shown in Fig.1). This case mainly analyzes optimization selection of risers. Therefore, under the premise of the same conditions, I designed three different kinds of risers' schemes (shown in Fig.2, Fig.3, Fig.4). Then I analyzed and compared the 
quality defect on the surface, and finally determined the optimal scheme.

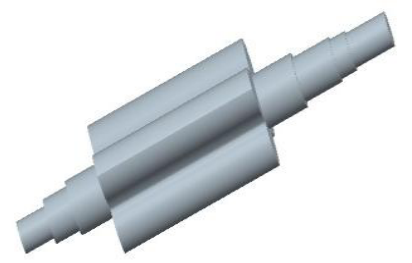

Figure 1. Three-dimension graph

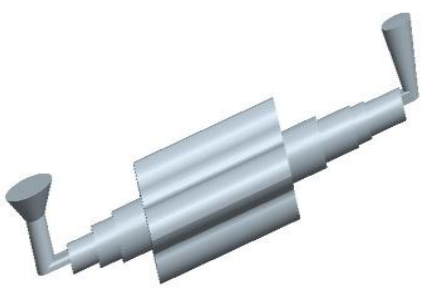

Figure 2. One-riser model

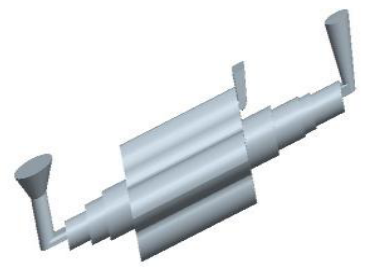

Figure 3. Two-riser model

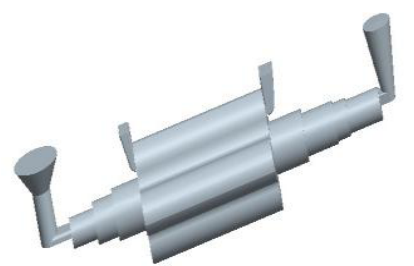

Figure 4. Three-riser model

According to the analysis results of Fig.5, Fig.6, entrapped air is considered. From a comparison of these two figures, it is clear that entrapped air is more in Fig. 5 which is a model of two rises; especially its red portions in the middle are more. This shows that entrapped air in Fig. 5 is more than the one which is the corresponding part in Fig. 6. Thus, we can see that the three-riser design is more reasonable when we consider entrapped air.

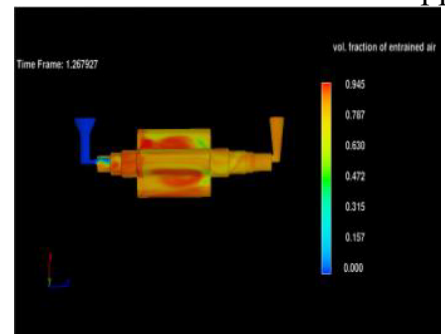

Figure 5. The entrapped air picture of one-riser model analysis

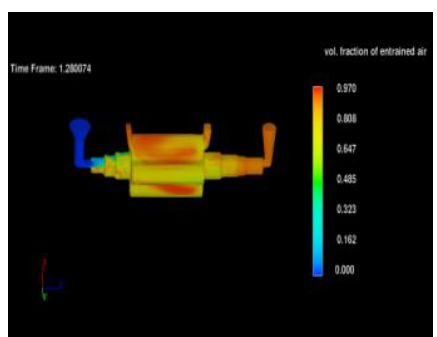

Figure 6. The entrapped air picture of three-riser model analysis

According to Fig.7, Fig.8 the analysis results, surface defect density is considered. From a comparison of these two figures, it is clear that surface defect density in the two-riser model in Fig.7 is lighter which the maximum value is only 14.997. While in Fig.8, the maximum of surface defect density in the three riser model is 37.32 . After comprehensive comparison, we can see that surface defects density of the three-riser model in Fig. 8 is more, and its effect is better. Thus surface defect density of the three riser design is more reasonable.

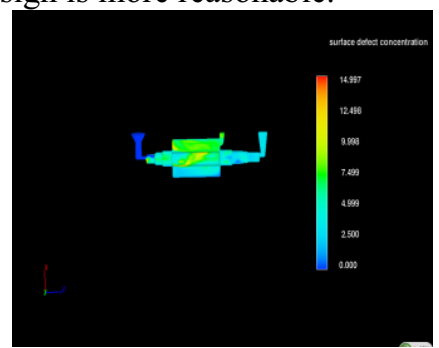

Figure 7. The surface defect density picture of two-rise model analysis

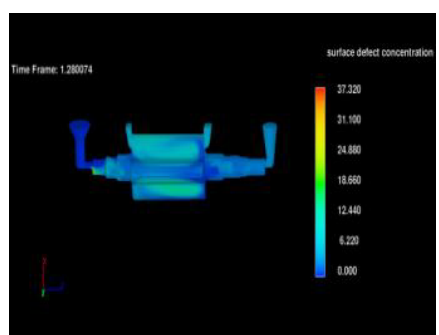

Figure 8. The surface defect density picture of three-rise model analysis

\section{Conclusions}

According to the analyses of the analysis different schemes what are mainly the different designs of risers which include one, two, and three risers. This text respectively carries on concrete analysis of entrapped air and surface defects density. Then it results in a detailed and visualized analysis. After comparing these two respects, the effect of the design with three rises is the best. Therefore, the final design is the one of three-riser. On the basis of the actual production that was carried by the result of simulation analysis, we can find out the effect of the chosed scheme is well. It can fulfill practical demands completely. Compared with other past schemes, not only can this scheme shorten planning time, but it can improve the quality of production. Therefore, it is worthy of applying simulation analysis to the production of this kind of screws. 


\section{Acknowledgments}

The authors acknowledge the support of science and technology project of Nanchang Municipal Science and Technology Bureau (analog simulation of outline generation and optimum value of blank finishing allowance of screw assembly --- the key components of screw expansion generators). And the support of science and technology project of Science and Technology Department of Jiangxi Province (grant Number 20133BBE50013)

\section{References}

1. Chen Liangle, Yu Yuefeng. 2012, "The Application of A Screw Expanding Power Generator in Recycling Waste Heat of Tunnel Kiln”. Tile, No.11: 69-71

2. Sun Ruixia, Liu Qiping, He Bingfeng, Wang Kuangfei, Mi Guofa. 2009, "The Application of Computer Simulation in Optimizing Large Steel-ingot Process " . Technology of Metal Casting and Forge Welding, Vol.38, No. 21:5659

3. Hu Zhijun, Yang Mingbo, Gong Xibing, Li Guorui. 2006, "Numerical Simulation Optimization of Casting Process". Science of Military Materials and Engineering Vol.29 No.6:51-53

4. Qian Yijun, Cheng Zhaohu, Yu Hao. 2012. "The Application Process of Numerical Simulation in Casting". Precise Forming Engineering. No. 07:39-43

5. Liu Baicheng. 2005, "The Tendency of Casting Technology and Computer Simulation". Casting Technology, Vol.26,No.7:611-617 